\title{
Outcomes in Seriously Head-Injured Patients Undergoing Pre-Hospital Tracheal Intubation vs. Emergency Department Tracheal Intubation ${ }^{*}$
}

\author{
John M. Tallon ${ }^{1 \#}$, Gordon Flowerdew ${ }^{2}$, Ronald D. Stewart ${ }^{3}$, George Kovacs ${ }^{4}$ \\ ${ }^{1}$ Departments of Emergency Medicine and Surgery, Capital Health, Dalhousie University, Halifax, Canada; ${ }^{2}$ Department of Commu- \\ nity Health and Epidemiology, Dalhousie University, Halifax, Canada; ${ }^{3}$ Faculties of Medicine and Health Professions, Dalhousie \\ University, Halifax, Canada; ${ }^{4}$ Department of Emergency Medicine, Dalhousie University, Halifax, Canada. \\ Email: \#jtallon@dal.ca
}

Received November $24^{\text {th }}, 2012$; revised December $24^{\text {th }}, 2012$; accepted January $3^{\text {rd }}, 2013$

\begin{abstract}
Background: The optimal treatment of major head injuries in the resuscitative phase of care post-injury has yet to be determined. This study measured the effect on mortality of pre-hospital intubation (PHI) vs. emergency department intubation (EDI) of patients suffering serious head injury. Methods: In the single emergency medical services system for this Canadian province, we used a population-based trauma database, conventional logistic regression (with and without the use of a propensity score to control for selection effect bias) to evaluate the effect of PHI vs. EDI on in-hospital mortality. Inclusion criteria were age $\geq 16$ years, serious head injury (Abbreviated Injury Score $\geq 3$, non-penetrating trauma) and resuscitative intubation (PHI or EDI). Results: Over 5 years, 283 patients (2000-2005) met inclusion criteria. Conventional unconditional logistic regression modelled on mortality with "PHI vs. EDI" as the intervention of interest showed an odds ratio of 2.015 (95\% CI 1.062 - 3.825) for improved survival if these patients were intubated in the emergency department rather than in the pre-hospital phase of care. A propensity score adjustment demonstrated a similar but more conservative point estimate (OR 1.727, 95\% CI: 0.993 - 3.004). Conclusions: This observational study demonstrated a survival advantage with EDI (versus PHI) in seriously head-injured patients in a mature, province-wide emergency medical services system.
\end{abstract}

Keywords: Trauma; Head Injury; Tracheal Intubation; Mortality; Emergency Medical Services; Emergency Medicine

\section{Introduction}

Injury remains the leading cause of death in Canada for those under age 45 years and the largest contributor to potential life years lost of any single disease process in Canada under the age of 70 years [1]. Within this population group, traumatic brain injury (TBI) is the most common cause of death, and its prevention and treatment remain distinct challenges for health care providers, public health organizations and society as a whole [2,3]. TBI is the primary contributor to overall injury morbidity and disability-adjusted life years, as are other central nervous system injuries, such as spinal cord injury [3], and is the primary injury in patients discharged to long-term care. As such, while the prevention of head injuries with various engineering, educational and enforcement methods is of paramount importance, the optimal post-injury care of patients suffering head injury also has clear implications

\footnotetext{
"There are no conflicts of interest by the authors in relation to this article. ${ }^{\#}$ Corresponding author.
}

for improved outcomes.

Secondary brain injury (after the primary traumatic insult) can be directly attributable to transient episodes of hypoxia and/or hypotension, either of which can double associated post-injury mortality [4]. Relatively simple pre-hospital interventions, including prevention of hypoxia and the use of fluids to prevent hypotension, can thus potentially ameliorate significant mortality and morbidity in the patient suffering head injury [4-6]. The emphasis on empiric and timely intervention with oxygen and tracheal intubation in the emergency medical services (EMS) phase of care has been continually emphasized in the literature and published in guidelines for care of this cohort of patients $[7,8]$. Tracheal intubation for seriously ill, head-injured patients has served as a resuscitative treatment tenet for decades in the pre-hospital environment [9-14]. However, it is only recently that this advanced intervention has been comprehensively studied to determine its true effectiveness in the field.

Recently a number of studies have questioned the 
benefit of pre-hospital tracheal intubation (PHI) and suggested possible harm. Recommendations are now being formulated for the first time proposing that this procedure be performed only in the more controlled environment of an emergency department or by critical care providers. In Canada, only one such study has been performed, and there are no reported urban or rural studies encompassing an entire state or province, and utilizing one pre-hospital system [15]. The Canadian province of Nova Scotia is unique in that it has a single pre-hospital system with a single province-wide trauma database, as well as standardized medical treatment protocols within a single ground and air ambulance system.

The main purpose of this study was to measure the effects of PHI vs. emergency department intubation (EDI) on the mortality outcome of seriously head injured patients in a modern province-wide Canadian pre-hospital or EMS system.

\section{Ethics Approval}

This study obtained full ethics approval from the Capital Health Ethics Review Board.

\section{Methods}

This was a retrospective cohort study using data from the Nova Scotia Trauma Program Registry, a province-wide registry that houses comprehensive data on all severely injured patients [16]. Over 5600 patients are now included in the trauma registry, which has detailed information on seriously injured patients in Nova Scotia dating back to 1994. Data sources for the registry include the in-hospital patient record, as well as the pre-hospital patient care record, which includes ground ambulance and/or air medical transport patient care records and information from the provincial communication and dispatch centre for EMS. The main outcome of interest was in-hospital mortality. The Canadian province of Nova Scotia had a population of 913,462 in 2005 and 908,007 in 2001, with minimal variation during the study period; it is approximately $55,284 \mathrm{~km}^{2}$ in size, with a significant rural component (53\%) [17]. A single tertiary care and neurosurgical centre for the entire province is located in the provincial capital of Halifax.

\subsection{Study Sample}

All subjects $\geq 16$ years of age and $<80$ years of age between April 1, 2000, and March 31, 2005, were included. $[18,19]$. Subjects must have suffered a serious blunt head injury with an associated Abbreviated Injury Scale (AIS) of $\geq 3$ for the head (neck excluded), and have been entered into the provincial trauma registry. The AIS scale number arithmetically defines serious head injury and has been used in other pre-hospital and trauma head injury research to define serious head injury [20-25]. Finally, included subjects must have undergone tracheal intubation either in the field (PHI) or in an emergency department (EDI). Patients transferred from another facility by ambulance were included if, during the transfer, they underwent intubation by EMS providers. If they were intubated in an emergency department prior to transport, they were included in the EDI group.

Excluded were those with penetrating injuries to the head (such as gunshot wounds), those with isolated major burns or those who were intubated at other phases of care (i.e. the operating room, intensive care unit, etc.).

Figure 1 shows the conceptual model for the study and final sample derivation methodology.

The final cohort included 329 eligible patients with major head injury who underwent PHI or EDI over the five-year period.

\subsection{Data Analysis}

All analyses were conducted using SPSS, version 15.0 (SPSS Inc., Chicago, IL). Epi Info version 3.3.2 (Centers for Disease Control and Prevention, Atlanta, GA) was used for sample-size calculations.

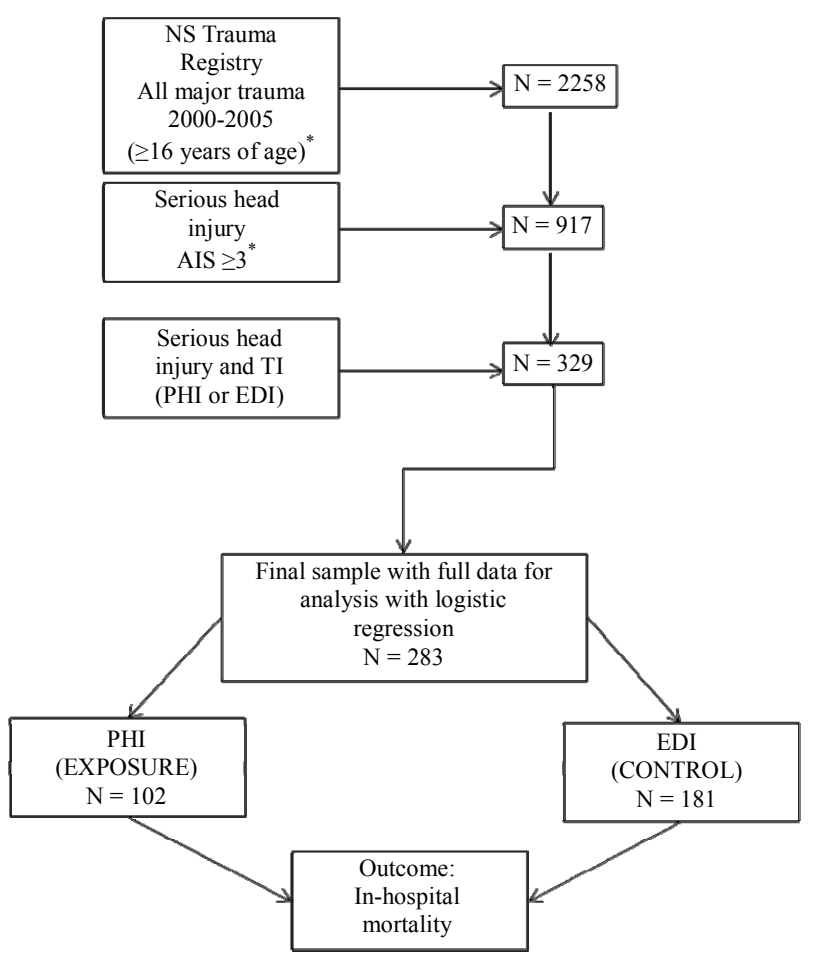

Figure 1. Study sample: 16 - 79 years of age. "Excluding deaths at scene. AIS: Abbreviated Injury Scale; EDI: emergency department intubation; PHI: pre-hospital intubation; TI: tracheal intubation. 
Descriptive statistics were used to explore the data prior to detailed analysis; this included means, medians, standard deviations, histograms and box plots for continuous data and tabulation and percentages for categorical data. Univariate analysis using the Chi-square test for categorical variables and the Mann-Whitney $U$ test for continuous variables was performed, with significance defined as a $p<0.05$. The association between the primary outcome variable (survival) and the predictor variables was also evaluated using univariate logistic regression analysis. This produced a crude odds ratio for the association between each predictor and the primary outcome.

Unconditional logistic regression by backwards likelihood ratio-based stepwise methodology was used to determine the effect of PHI (vs. EDI) on outcome, controlling for identified confounding variables [26,27]. Confounders included in the initial model evaluation were: age, injury severity score, presence of pre-hospital shock, Glasgow Coma Score (GCS) at the scene of injury as assessed by EMS, surgical intervention in the operating room, presence of co-morbidities and total time to final emergency department arrival from EMS arrival at the injury scene. Odds ratios for survival were generated with $95 \%$ confidence intervals.

A propensity score was then derived by generating individual predicted probabilities of PHI for each subject in the original non-parsimonious logistic regression mod- el [28], with all potential cofounders included. The mod- el selected by stepwise regression was then rerun, with the propensity score added as a predictor.

\section{Results}

Between April 1, 2000, and March 31, 2005, 2258 patients with major injuries entered into the Nova Scotia Trauma Registry. Of these patients, 917 (40.6\%) suffered major head injury as defined by an AIS of $\geq 3$.

Of those suffering a major head injury, $329(35.9 \%$ of major head injuries) underwent either PHI $(\mathrm{n}=116$, $35.3 \%$ of intubations) or EDI ( $\mathrm{n}=213,64.7 \%$ of intubations). This represents a rate of 65.8 patients with major head injury intubated per year during the study period. Of the 329 patients with major head injury undergoing tracheal intubation, 283 subjects had complete data for use in the logistic regression analysis (see Figure 1).

Table 1 summarizes the characteristics of the two study populations. Overall, the PHI group had a significantly higher unadjusted mortality, higher injury severity score (ISS), lower GCS (at scene), a shorter length of stay and a higher prevalence of hypotension (shock) overall, indicating a different and more severely injured cohort. Unadjusted mortality in the EDI cohort was $24.9 \%$, and in the PHI cohort was $50 \%$. These numbers are quite similar to those found by Wang [25], who demonstrated an overall unadjusted mortality of $37.1 \%$, with higher mortality in the PHI cohort $(48.5 \%)$ vs. his EDI cohort (28.2\%).

The mechanisms of injury for the final head injury cohort were characterized by a preponderance of motor vehicle collisions $(52.6 \%)$, as would be expected, followed by falls (25.8 \%) (Table 2). Overall crude mortality in intubated patients was $33.8 \%$, demonstrating thatthis was a seriously injured population.

Table 1. Characteristics of study cohort $(n=329)$.

\begin{tabular}{|c|c|c|c|}
\hline Characteristic & PHI $(n=116)$ & $\operatorname{EDI}(n=213)$ & p value \\
\hline Mortality, \% (n) & $50(58)$ & $24.9(53)$ & $\mathrm{p}<0.001^{*}$ \\
\hline ISS median (mean) & $29(32.3)$ & $26(27.7)$ & $\mathrm{p}=0.003^{\dagger}$ \\
\hline GCS at scene median (mean) & $4(5.9)$ & $8(8.3)$ & $\mathrm{p}<0.001^{\dagger}$ \\
\hline ED SBP, mmHg median (mean) & $130(118.5)$ & $138(137.7)$ & $\mathrm{p}=0.009^{\dagger}$ \\
\hline Age median (mean) & $34.5(39.2)$ & $38(40.2)$ & $\mathrm{NS}^{\dagger}$ \\
\hline Sex, $\%$ male $(n)$ & $75.9(88)$ & $72.3(154)$ & $\mathrm{NS}^{*}$ \\
\hline LOS, days, median (mean) & $6(22)$ & $12(28.9)$ & $\mathrm{p}=0.003^{\dagger}$ \\
\hline Total time to final ED, min, median (mean) & $105(155)$ & $161.5(205.2)$ & $\mathrm{NS}^{\dagger}$ \\
\hline Comorbidities present, $\%(\mathbf{n})$ & $26.7(31)$ & $31.0(\mathbf{6 6})$ & $\mathrm{NS}^{*}$ \\
\hline Number of intermediate hospitals, $\%$ & & & $\mathrm{p}=0.001^{*}$ \\
\hline 0 & $59(68)$ & $39(83)$ & \\
\hline 1 & $39.3(46)$ & $53.5(114)$ & \\
\hline 2 & $1.7(2)$ & $7.5(16)$ & \\
\hline Presence of shock, ${ }^{\ddagger} \%$ (n) & $17.0(\mathbf{2 0})$ & $4.9(\mathbf{1 0})$ & $\mathrm{p}=0.001^{*}$ \\
\hline OR intervention, \% (n) & $47.4(55)$ & $54.9(\mathbf{1 1 7})$ & $\mathrm{NS}^{*}$ \\
\hline
\end{tabular}

Data are median (mean) unless otherwise specified; ${ }^{*}$ Chi-square test; ${ }^{\dagger}$ Mann-Whitney U Test; ${ }^{\dagger}$ Systolic blood pressure < 90 mm Hg; ED, emergency department; EDI, emergency department intubation; ED SBP, emergency department systolic blood pressure; GCS, Glasgow Coma Score; ISS, injury severity score; LOS, length of stay; NS, not significant; OR, operating room; PHI, pre-hospital intubation. 


\subsection{Univariate Analysis}

Table 3 shows the characteristics of the study population based upon the primary outcome measure (mortality). Patients who survived had a statistically lower ISS, higher GCS, lower age, longer length of stay, longer time to final emergency department arrival, less shock and more frequent operating room surgical interventions.

Further univariate analysis, using simple logistic regression to elaborate the crude relationship between mortality and the study intervention (PHI vs. EDI) showed significant odds of survival if intubation was performed in the emergency department environment (OR 3.019, 95\% CI 1.871 - 4.871). A similar survival relationship was demonstrated when analyzing outcomes of air ambulance intubation vs. ground ambulance intubation (OR 6.316, 95\% CI 2.718 - 14.672), although the wide confidence interval was reflective of the small air medical sample size $(n=45)$. No statistical difference was shown between air ambulance and emergency department intubation outcomes in univariate simple logistic regression analysis (OR 1.018, 95\% CI $0.482-2.148$ ).

\subsection{Multivariate Analysis}

Prediction of mortality predicated upon conventional logistic regression was performed by the inclusion of co- variates felt to be potential confounders previously described in the literature and available within the provincial database. These final chosen covariates (as well as the "treatment/intervention" variable) included GCS, shock, ISS, age, operating room surgical intervention, comorbidities and total time to final emergency department. We then performed conventional unconditional logistic regression using a backwards stepwise likelihood ratio selection process.

The total number of patients in the database available for this analysis (and subsequent propensity analysis and adjustment) was 283 (or $86 \%$ of all subjects); the remaining subjects (46/14\%) were unavailable for analysis due to missing data elements. This final analysis cohort

Table 2. Mechanism of injury in study cohort.

\begin{tabular}{lcc}
\hline & Frequency & \% \\
\hline Motor vehicle accident & 173 & 52.6 \\
Fall & 85 & 25.8 \\
Assault & 26 & 7.9 \\
Pedestrian vs. vehicle & 23 & 7.0 \\
Other & 22 & 6.7 \\
Total & 329 & 100.0 \\
\hline
\end{tabular}

Table 3. Characteristics of survivors and non-survivors $(n=329)$.

\begin{tabular}{|c|c|c|c|}
\hline & Survivors $(n=218)$ & Non-survivors $(\mathrm{n}=111)$ & p value \\
\hline ISS median (mean) & $26.0(27.4)$ & $29.0(33)$ & $\mathrm{p}=0.001^{\dagger}$ \\
\hline GCS scene $(\mathrm{n}=301)$ median (mean) & $8.0(8.3)$ & $3.0(5.8)$ & $\mathrm{p}<0.001^{\dagger}$ \\
\hline ED SBP, $\operatorname{mmHg}(\mathrm{n}=314)$ median (mean) & $136(135.4)$ & $130(121.5)$ & $\mathrm{p}=0.179$ \\
\hline Age, y $(n=329)$ median (mean) & $32(36)$ & $48(48.2)$ & $\mathrm{p}<0.001^{\dagger}$ \\
\hline Sex, male $(n=242) ; \%(n)$ & $74(161)$ & $73(81)$ & $\mathrm{p}=0.864^{*}$ \\
\hline LOS, $d(n=327)$ mean (median) & $23(36.4)$ & $1.0(6.6)$ & $\mathrm{p}<0.001^{\dagger}$ \\
\hline $\begin{array}{l}\text { Total time to final ED, } \min (n=307) \\
\text { Mean (median) }\end{array}$ & $160.5(200.1)$ & $72.0(162)$ & $\mathrm{p}=0.028^{\dagger}$ \\
\hline Comorbidities present, $\%(n) ;(n=97)$ & $17.3(57)$ & $12.2(40)$ & $\mathrm{p}=0.063^{*}$ \\
\hline \# intermediate hospitals, $\%,(n)$ & & & $\mathrm{p}=0.056^{*}$ \\
\hline 0 & $70(153)$ & $54.6(61)$ & \\
\hline 1 & $24.5(53)$ & $40(44)$ & \\
\hline 2 & $5.5(12)$ & $5.4(6)$ & \\
\hline Presence of shock, ${ }^{\ddagger} \%(n),(n=29)$ & $1.9(6)$ & $7.3(23)$ & $\mathrm{p}<0.001^{*}$ \\
\hline OR intervention, \% (n) $(\mathrm{n}=172)$ & $41.6(137)$ & $10.6(35)$ & $\mathrm{p}<0.001^{*}$ \\
\hline
\end{tabular}

Data are median (mean) unless otherwise specified; ${ }^{*}$ Chi-square test; $\uparrow$ Mann-Whitney U Test; $\downarrow$ Systolic blood pressure $<90$ mm Hg; ED, emergency department; EDI, emergency department intubation; ED SBP, emergency department systolic blood pressure; GCS, Glasgow Coma Score; ISS, injury severity score; LOS, length of stay; NS, not significant; OR, operating room; PHI, pre-hospital intubation. 
was comprised of $n=102$ PHI subjects and $n=181$ EDI subjects (see Figure 1).

The results of the multivariate analysis showed that EDI was associated with an odds ratio of $2.015(95 \% \mathrm{CI}$ 1.062 - 3.825), indicative of an improved probability of survival vs. PHI in seriously head-injured patients within the parameters of this model.

The outcome and intervention model was re-run with the incorporation of a propensity score comprised of all confounders originally included in the non-parsimonious model. Propensity scoring adjustment has been well established for use in observational clinical studies to assist in reducing bias associated with the intervention of interest. The propensity score will most often render a more conservative outcome than traditional logistic regression modelling. In this study, the use of a propensity score resulted in an odds ratio demonstrating a more conservative (but still positive) point estimate (OR 1.727, $95 \%$ CI $0.993-3.004$ ), but with a confidence interval not clearly favouring improved survival for EDI.

\section{Discussion}

Controversy surrounds the issue of optimal airway management in the resuscitative and transport phase of patients suffering severe head injury. Whether a patient should undergo tracheal intubation in the field (by EMS personnel) or rapid transfer and intubation in an emergency department is of considerable importance considering the impact on head-injury outcomes of timely and optimal oxygenation and ventilation clearly have on head injury outcomes.

Tracheal intubation for patients with severe head injuries has served as a basic treatment paradigm in the prehospital phase of care for decades in North America [2931] as well as Europe, Australia and New Zealand. Preventing hypoxia, optimizing gas exchange and protecting against aspiration with tracheal intubation constitute the foundation of resuscitative interventions in this critically ill group. However only recently has tracheal intubation been intensively studied to determine efficacy and effectiveness and to define indications for the procedure. There are now several studies that have questioned the assumed benefit of pre-hospital tracheal intubation, some even suggesting possible harm unless it is performed in the receiving emergency department or in very specialized pre-hospital environments, such as air medical transport [15,32-34].

Initial studies of PHI (primarily observational, single site, case series) were positive in their evaluations and outcomes analyses [35-39]. Christensen et al. in a Danish observational study, found an $8 \%$ survival improvement with PHI, but only if done by field anaesthesiologists as per the EMS care model in Denmark [35]. Ochs et al. [37] in a 2002 prospective case series, and then Davis et al. [36] following up on Ochs' work, demonstrated success with PHI in a severely head injured population performed by paramedics, but in these studies, "success" was defined procedurally; patient-oriented outcomes were not measured. An observational study (Sloane et al.) of patients undergoing PHI by aeromedical crews vs. major trauma patients brought to the emergency department for intubation found no difference in 30 day mortality between the two groups, but used only univariate analysis, and the two patient populations were significantly different [38]. Winchell and Hoyt [39] found that out-ofhospital endotracheal intubation was associated with a positive effect on traumatic brain injury survival and no effect on discharge destination, but again, their analysis used only univariate techniques without controlling for severity of injury or clinical confounders.

In the last few years, methodologically sophisticated and larger studies of PHI began, and more recent papers have produced results and conclusions that question the basic paradigm of pre-hospital resuscitation [15,20,21,24, 25,33,40-43]. For example, Murray et al. [24] using multivariate analysis and matching in an urban study using retrospective data from a trauma registry, found a significantly higher relative risk of mortality for the PHI group. Similarly, Bochicchio et al. [20] described a 1.85 times greater risk of death if intubation occurred in the field vs. the emergency department; it should be noted, however, that in this study the patients in the pre-hospital group suffered more severe injuries.

Two of the more recent and well-designed studies used mortality [21] and mortality/neurological function [25] as outcome measures in head-injured populations. In these seminal papers, outcome measures were statistically significantly better in the EDI groups than in the PHI groups. Davis et al. [21] used a prospective cohort design with historical controls, whereas Wang et al. [25] used a large urban retrospective trauma database and employed a propensity score adjustment to account for potential effects of pre-existing conditions, in-hospital complications and social factors. Although these two papers are methodologically quite different, their results were similar: Davis et al. concluded that PHI was associated with increased mortality and a decrease in good functional outcomes, while Wang et al. reported an increased adjusted odds of death and poor neurological outcome with PHI vs. EDI $[21,25]$. In a follow-up study using different methodology from their earlier paper, Davis et al. studied the association between PHI and EDI in severe head injury using a county-wide (San Diego) trauma registry and multivariate adjustment, and found a higher mortality (OR 2.1, 95\% CI 1.8 - 2.5) in the PHI group, confirming 
the findings of Wang et al. [23].

Only one Canadian study has been published evaluating the effects and outcomes of PHI in head-injured patients [34]. The Ontario Pre-hospital Advanced Life Support Study (OPALS III) studied the effect of advanced EMS life support interventions (including PHI) in 2867 major trauma patients (not only head injury) in a prospective before-and-after study. This study showed that system-wide implementation of full advanced life-support programs for EMS did not decrease mortality or morbidity [34]. The Wang study [25,37] also showed poor functional neurological outcomes in the PHI cohort, an outcome that could not be measured with the available data in the current study. No other study has encompassed an entire state or province or included rural and urban locations in one emergency medical services system.

In crude analysis for the current observational study, a distinctly unfavourable outcome (increased mortality) was statistically associated with PHI in a cohort containing more seriously injured than the EDI cohort, and this result was noted even after adjustment for injury severity, comorbidities and other prognostic variables. In this way, PHI demonstrated a worrisome poorer outcome, although a more conservative point estimate was obtained when a propensity score was utilized. These overall results of deleterious outcomes with PHI echo those of several other recent studies [20,22,34]. Unadjusted mortality in other studies ranged from $23 \%$ to $33 \%$ for PHI cohorts [20,21]; Wang et al. found a $48.5 \%$ unadjusted mortality in the PHI group, vs. $28.2 \%$ in the EDI group [25], rates that compare closely to those of the current study (PHI $50 \%$ mortality and EDI $24.9 \%$ mortality). Clearly, this cohort of patients (AIS $\geq 3$ ) contains patients with severe injuries and in need of advanced and timely interventions; the ongoing questions continue to be which intervention, and when and where?

The reasons for compromised outcomes with PHI in major head injury remain elusive but probably include issues involving paramedic training, the challenges of airway management skills maintenance, post-intubation ventilation often characterized by hyper- or hypoventilation, and prolonged intubation attempts with unrecognized hypoxia. It is assumed that these contributory factors are obviated by intubation in the more controlled environment of the emergency department (EDI).

Our findings of poor mortality outcomes with PHI are in the context of a large (province-wide) geographic area with all the challenges of urban and rural geography, time and distance $[21,25]$. The fact that we showed similar results in a more diverse EMS environment further informs the argument against PHI unless performed by advanced airway practitioners in a air medical or critical care settings.

\section{Limitations and Strengths}

Because this was a retrospective database analysis, it can demonstrate only association; to truly demonstrate causation, a prospective randomized trial of PHI vs. EDI would be required. As well, our study contains fewer patients than that reported in other studies [25], although sample-size calculations implied robust power to perform analysis and generate sound results. Although clear markers of neurological outcome would have been a significant addition to this study, limitations of the available database and lack of access to original charts and longterm follow-up meant that we were unable to determine meaningful clinical outcomes (such as neurological-function) other than mortality.

Another limitation is that the propensity score adjusts for confounding only by factors used in the model to generate propensity scores. There may be unobserved confounders that were missed. As well, propensity scores work better with larger sample sizes [28,44]. The fact that up to $14 \%$ of the overall cohort had some missing (and non-retrievable) data elements is also a concern if "missingness" is related to both the predictor of interest and the outcome.

Despite these limitations, the unique structure of the EMS system in Nova Scotia - one neurosurgical site for serious head injury as part of a single tertiary care centre for the entire province, plus the population-based mandate of the Nova Scotia trauma registry - means that this study possesses strengths of design not present in other published papers, which were primarily urban in nature and not state- or province-wide.

\section{Conclusion}

This study demonstrates a survival advantage for serious traumatic brain injury if intubation is performed in the emergency department rather than in the EMS environment when modelled with logistic regression (OR 2.015, $95 \%$ CI 1.062 - 3.825); this result was tempered by a more conservative point estimate when a propensity score adjustment was utilized (OR 1.727, 95\% CI 0.993 3.004). The limitations of a non-randomized design must be acknowledged, even when a propensity score is used. However, it seems unlikely that unobserved confounders would be responsible for all of the apparent association. This study provides evidence of association that contributes to the important ongoing debate concerning prehospital intubation in the injured patient.

\section{Acknowledgements}

The authors would like to acknowledge Ms. Corinne DeMone for her assistance with the manuscript. 


\section{REFERENCES}

[1] Public Health Agency of Canada, "Injury Surveillance OnLine," 2009.

http://dsol-smed.hc-sc.gc.ca/dsol-smed/is-sb/index-eng.php

[2] J. A. Langlois, W. Rutland-Brown and K. E. Thomas, "Traumatic Brain Injury in the United States: Emergency Department Visits, Hospitalizations, and Deaths," Department of Health and Human Services (US), Centers for Disease Control and Prevention, National Center for Injury Prevention and Control, Atlanta, 2004.

http://www.cdc.gov/ncipc/pub-res/tbi_in_us_04/TBI_Inci dents.htm

[3] D. J. Thurman, C. Alverson, K. A. Dunn, et al., "Traumatic Brain Injury in the United States: A Public Health Perspective," Journal of Head Trauma Rehabilitation, Vol. 14, No. 62, 1999, pp. 602-615. doi:10.1097/00001199-199912000-00009

[4] R. M. Chesnut, L. F. Marshall and M. R. Klauber, "The Role of Secondary Brain Injury in Determining Outcome from Severe Head Injury," Journal of Trauma, Vol. 34, No. 2, 1993, pp. 216-222. doi:10.1097/00005373-199302000-00006

[5] J. H. Chi, M. M. Knudson, M. J. Vassar, et al., "Prehospital Hypoxia Affects Outcome in Patients with Traumatic Brain Injury: A Prospective Multicenter Study," Journal of Trauma, Vol. 61, No. 5, 2006, pp. 1134-1141. doi:10.1097/01.ta.0000196644.64653.d8

[6] P. A. Jones, P. J. Andrews, S. Midgley, et al., "Measuring the Burden of Secondary Insults in Head-Injured Patients during Intensive Care," Journal of Neurosurgical Anesthesiology, Vol. 6, No. 1, 1994, pp. 4-14.

[7] N. Badjatia, N. Carney, T. J. Crocco, et al., "Guidelines for Prehospital Management of Traumatic Brain Injury," Prehospital Emergency Care, Vol. 12, No. 1, 2008, pp. S1-S52.

[8] R. M. Chesnut, "Care of Central Nervous System Injuries," Surgical Clinics of North America, Vol. 87, No. 1, 2007, pp. 119-156. doi:10.1016/j.suc.2006.09.018

[9] A. M. Brambrink and I. P. Koerner, "Prehospital Advanced Trauma Life Support: How Should We Manage the Airway, and Who Should Do It?" Critical Care, Vol. 8, No. 1, 2004, pp. 3-5. doi:10.1186/cc2420

[10] E. M. Bulger, M. K. Copass, R. V. Maier, et al., "An Analysis of Advanced Prehospital Airway Management," Journal of Emergency Medicine, Vol. 23, No. 2, 2002, pp. 183-189. doi:10.1016/S0736-4679(02)00490-0

[11] S. Pearson, "Comparison of Intubation Attempts and Completion Times before and after the Initiation of a Rapid Sequence Intubation Protocol in an Air Medical Transport Program," Air Medical Journal, Vol. 22, No. 6, 2003, pp. 28-33. doi:10.1016/j.amj.2003.08.004

[12] P. E. Pepe, M. K. Copass and T. H. Joyce, "Prehospital Endotracheal Intubation: Rationale for Training Emergency Medical Personnel," Annals of Emergency Medicine, Vol. 14, No. 11, 1985, pp. 1085-1092. doi:10.1016/S0196-0644(85)80927-6

[13] R. F. Sing, M. F. Rotondo, D. H. Zonies, et al., "Rapid
Sequence Induction for Intubation by an Aeromedical Transport Team: A Critical Analysis," American Journal of Emergency Medicine, Vol. 16, No. 6, 1998, pp. 598602. doi:10.1016/S0735-6757(98)90227-3

[14] N. Stocchetti, A. Furlan and F. Volta, "Hypoxemia and Arterial Hypotension at the Accident Scene in Head Injury," Journal of Trauma, Vol. 40, No. 5, 1996, pp. 764767. doi:10.1097/00005373-199605000-00014

[15] B. J. Zink and R. F. Maio, "Out-of-Hospital Endotracheal Intubation in Traumatic Brain Injury: Outcomes Research Provides Us with an Unexpected Outcome," Annals of Emergency Medicine, Vol. 44, No. 5, 2004, pp. 451-453. doi:10.1016/j.annemergmed.2004.05.001

[16] Trauma.org, 2009. http://www.trauma.org

[17] Vital Statistics. Access Nova Scotia, 2009. http://www.gov.ns.ca/snsmr/vstat

[18] B. J. Gabbe, P. A. Cameron, R. Wolfe, et al., "Predictors of Mortality, Length of Stay and Discharge Destination in Blunt Trauma," ANZ Journal of Surgery, Vol. 75, No. 8, 2005, pp. 650-656. doi:10.1111/j.1445-2197.2005.03484.x

[19] D. D. Trunkey, R. M. Cahn, B. Lenfesty, et al., "Management of the Geriatric Trauma Patient at Risk of Death: Therapy Withdrawal Decision Making," Archives of Surgery, Vol. 135, No. 1, 2000, pp. 34-38. doi:10.1001/archsurg.135.1.34

[20] G. V. Bochicchio, O. Ilahi, M. Joshi, et al., "Endotracheal Intubation in the Field Does Not Improve Outcome in Trauma Patients Who Present without an Acutely Lethal Traumatic Brain Injury," Journal of Trauma, Vol. 54, No. 2, 2003, pp. 307-311. doi:10.1097/01.TA.0000046252.97590.BE

[21] D. P. Davis, D. B. Hoyt, M. Ochs, et al., "The Effect of Paramedic Rapid Sequence Intubation on Outcome in Patients with Severe Traumatic Brain Injury," Journal of Trauma, Vol. 54, No. 3, 2003, pp. 444-453. doi:10.1097/01.TA.0000053396.02126.CD

[22] D. P. Davis, J. Peay, J. A. Serrano, et al., "The Impact of Aeromedical Response to Patients with Moderate to Severe Traumatic Brain Injury," Annals of Emergency Medicine, Vol. 46, No. 2, 2005, pp. 115-122. doi:10.1016/j.annemergmed.2005.01.024

[23] D. P. Davis, J. Peay, M. J. Sise, et al., "The Impact of Prehospital Endotracheal Intubation on Outcome in Moderate to Severe Traumatic Brain Injury," Journal of Trauma, Vol. 58, No. 5, 2005, pp. 933-939. doi:10.1097/01.TA.0000162731.53812.58

[24] J. A. Murray, D. Demetriades, T. V. Berne, et al., "Prehospital Intubation in Patients with Severe Head Injury," Journal of Trauma, Vol. 49, No. 6, 2000, pp. 1065-1070. doi:10.1097/00005373-200012000-00015

[25] H. E. Wang, A. B. Peitzman, L. D. Cassidy, et al., "Outof-Hospital Endotracheal Intubation and Outcome after Traumatic Brain Injury," Annals of Emergency Medicine, Vol. 44, No. 5, 2004, pp. 439-450. doi:10.1016/j.annemergmed.2004.04.008

[26] F. E. Harrell Jr., K. L. Lee and D. B. Mark, "Multivari- 
able Prognostic Models: Issues in Developing Models, Evaluating Assumptions and Adequacy, and Measuring and Reducing Errors," Statistics in Medicine, Vol. 15, No. 4, 1996, pp. 361-387. doi:10.1002/(SICI)1097-0258(19960229)15:4<361::AIDSIM168>3.0.CO;2-4

[27] E. W. Steyerberg, M. J. Eijkemans, F. E. Harrell Jr., et al., "Prognostic Modeling with Logistic Regression Analysis: In Search of a Sensible Strategy in Small Data Sets," Medical Decision Making, Vol. 21, No. 1, 2001, pp. 45-56. doi:10.1177/0272989X0102100106

[28] C. D. Newgard, J. R. Hedges, M. Arthur, et al., "Advanced Statistics: The Propensity Score-A Method for Estimating Treatment Effect in Observational Research," Academic Emergency Medicine, Vol. 11, No. 9, 2004, pp. 953-961.

[29] American College of Surgeons, "American College of Surgeons Committee on Trauma Advanced Trauma Life Support Instructor Manual," First Impressions, Chicago, 2004.

[30] National Association of Emergency Medical Technicians (NAEMT), "Airway and Ventilation. Prehospital Trauma Life Support," Mosby Elsevier, St Louis, 2007.

[31] F. Procaccio, N. Stocchetti, G. Citerio, et al., "Guidelines for the Treatment of Adults with Severe Head Trauma (Part I). Initial Assessment; Evaluation and Pre-Hospital Treatment; Current Criteria for Hospital Admission; Systemic and Cerebral Monitoring," Journal of Neurosurgical Sciences, Vol. 44, No. 1, 2000, pp. 1-10.

[32] D. P. Davis, "Should Invasive Airway Management Be Done in the Field?" Canadian Medical Association Journal, Vol. 178, No. 9, 2008, pp. 1171-1173. doi:10.1503/cmaj.080234

[33] J. D. Nolan, "Prehospital and Resuscitative Airway Care: Should the Gold Standard Be Reassessed?" Current Opinion in Critical Care, Vol. 7, No. 6, 2001, pp. 413-421. doi:10.1097/00075198-200112000-00008

[34] G. Stiell, L. P. Nesbitt, W. Pickett and OPALS Study Group, "The OPALS Major Trauma Study: Impact of Advanced Life-Support on Survival and Morbidity," Canadian Medical Association Journal, Vol. 178, No. 9, 2008, pp. 1141-1152. doi:10.1503/cmaj.071154

[35] E. F. Christensen and C. C. Hoyer, "Prehospital Tracheal Intubation in Severely Injured Patients: A Danish Observational Study," British Medical Journal, Vol. 327, No.
7414, 2003, pp. 533-534. doi:10.1136/bmj.327.7414.533

[36] D. P. Davis, M. Ochs, D. B. Hoyt, et al., "ParamedicAdministered Neuromuscular Blockade Improves Prehospital Intubation Success in Severely Head-Injured Patients," Journal of Trauma, Vol. 55, No. 4, 2003, pp. 713719. doi:10.1097/01.TA.0000037428.65987.12

[37] M. Ochs, D. Davis, D. Hoyt, et al., "Paramedic-Performed Rapid Sequence Intubation of Patients with Severe Head Injuries," Annals of Emergency Medicine, Vol. 40, No. 2, 2002, pp. 159-167. doi:10.1067/mem.2002.126397

[38] C. Sloane, G. M. Vilke, T. C. Chan, et al., "Rapid Sequence Intubation in the Field versus Hospital in Trauma Patients," Journal of Emergency Medicine, Vol. 19, No. 3, 2000, pp. 259-264. doi:10.1016/S0736-4679(00)00235-3

[39] R. J. Winchell and D. B. Hoyt, "Endotracheal Intubation in the Field Improves Survival in Patients with Severe Head Injury, Trauma Research and Education Foundation of San Diego," Archives of Surgery, Vol. 132, No. 6, 1997, pp. 592-597. doi:10.1001/archsurg.1997.01430300034007

[40] V. Dunford, D. P. Davis, M. Ochs, et al., "Incidence of Transient Hypoxia and Pulse Rate Reactivity during Paramedic Rapid Sequence Intubation," Annals of Emergency Medicine, Vol. 42, No. 6, 2003, pp. 721-728. doi:10.1016/S0196-0644(03)00660-7

[41] M. Gausche, R. J. Lewis, S. J. Stratton, et al., "Effect of Out-of-Hospital Pediatric Endotracheal Intubation on Survival and Neurological Outcome: A Controlled Clinical Trial," Journal of the American Medical Association, Vol. 283, No. 6, 2000, pp. 783-790. doi:10.1001/jama.283.6.783

[42] M. Ochs, D. P. Davis and D. B. Hoyt, "Lessons Learned during the San Diego Paramedic RSI Trial," Journal of Emergency Medicine, Vol. 24, No. 3, 2003, pp. 343-344. doi:10.1016/S0736-4679(03)00006-4

[43] H. E. Wang, D. F. Kupas, P. M. Paris, et al., "Preliminary Experience with a Prospective, Multi-Centered Evaluation of Out-of-Hospital Endotracheal Intubation," Resuscitation, Vol. 58, No. 1, 2003, pp. 49-58. doi:10.1016/S0300-9572(03)00058-3

[44] D. B. Rubin. "Estimating Causal Effects from Large Data Sets Using Propensity Scores," Annals of Internal Medicine, Vol. 127, No. 8, 1997, pp. 757-763. 